\title{
HIGH SENSITIVITY MAGNETIC SENSOR CONCEPT BASED ON MAGNETOSTRICTIVELY INDUCED PERTURBATIONS IN PLATE RESONATOR CHARACTERISTICS
}

\author{
Gokhan Hatipoglu and Srinivas Tadigadapa \\ Department of Electrical Engineering, The Pennsylvania State University, University Park, USA
}

\begin{abstract}
Integrated, micromachined, room temperature magnetic sensors and sensor arrays with high sensitivity are highly desirable for several applications including magnetoencephalography, magnetic resonance imaging etc. In this work, we propose a sensor consisting of a micromachined AT-cut quartz bulk-acoustic wave cantilever resonator $(500 \times 45 \times 22 \mu \mathrm{m})$ with one of the electrodes having $300 \mathrm{~nm}$ thick magnetostrictive Metglas ${ }^{\circledR}$ layer. The perturbations induced in the quartz resonator due to magnetically induced deformation in the quartz-Metglas ${ }^{\circledR}$ unimorph structure results a shift in the fundamental thickness-shear frequency and the at resonance impedance characteristics of the resonator. Using this configuration we have been able to achieve magnetic field sensitivity in the microTesla range with the possibility of extending the sensitivity into nanoTesla levels. This paper explores the concept using magnetostrictively induced perturbations in the plate resonator characteristics for magnetic sensing applications.
\end{abstract}

\section{INTRODUCTION}

A variety of chip scale magnetic sensors covering several orders of magnitude of sensitivity from milliTesla to femtoTesla fields have been developed and demonstrated [1-4]. However, sensors that can measure the picoTesla and femtoTesla range magnetic fields either operate at cryogenic temperatures such as SQUID sensors [5, 6] or use alkali atom vapors and optical detection [7]. For biomedical imaging applications such as magnetoencephalography, magnetic sensors with sensitivity better than the pico-Tesla range are required $[6,8]$. Thus there is a clear opportunity to develop competing sensor technology that is compact, operates at room temperature, has the required highsensitivity and a good price-to-performance ratio [9]. Therefore, integrated and micromachined, room temperature magnetic sensors and sensor arrays with high sensitivity are highly desirable for these kind of applications. Previously, we have reported two different magnetic sensor technologies: i) magnetoelectric flexural gate transistors with DC magnetic field sensitivity of $60 \mathrm{nT} / \sqrt{\mathrm{Hz}}$ [10] ii) quartz crystal/ferrofluid magnetic sensors employing magnetoviscous effect with DC magnetic field sensitivity of 15 $\mathrm{nT} \sqrt{\mathrm{Hz}}$ [11]. Here, we investigate a promising magnetoelectric magnetic sensor concept consisting of quartz crystal microcantilever resonator coupled to a Metglas ${ }^{\circledR}$ magnetostrictive thin film.

Many ferromagnetic materials experience structural/shape change under applied magnetic fields - the phenomenon is known as magnetostriction. When such a thin film is placed on a piezoelectric film, the resulting strain due to magnetostriction is readily transduced into a charge by the piezoelectric material which can then be readout as a signal. This approach allows for the combination of high performance piezoelectric and magnetostrictive films to form high performance magnetoelectric magnetic sensing and has recently been extensively investigated [12-15]. However, until now these sensing modalities have not explicitly tried to exploit the effect of magnetostrictive strain on the resonance characteristics of piezoelectric crystal resonators. One can find reports on early stability studies on quartz resonators where sensitivity to magnetic fields was observed in resonators packaged and harnessed using ferromagnetic clamps. However, the overall sensitivity in these cases was extremely small $\sim 15 \mathrm{mHz} / \mathrm{mT}$ [16]. Of course in precision frequency applications the focus of these studies was to minimize any such magnetic field related effects. In this work, however, instead of considering frequency shift due to magnetostriction as a source of instability, the authors aim to exploit the phenomenon as a very sensitive magnetometer concept. This is achieved by combining a mechanically compliant, high frequency quartz cantilever resonator with a highly magnetostrictive material, Metglas ${ }^{\circledR}$, as one of the electrodes.

\section{MAGNETOSTRICTION \& MAGNETIC THIN FILMS}

Application of magnetic fields to magnetostrictive materials causes strain and stress along the material due to a change in the magnetization, which is known as the Joule (Direct) effect. It is also known that application of stresses to magnetostrictive materials induces domain magnetization changes, which is known as the Villari (Converse) effect. The constitutive equations for direct and converse magnetostrictive effects are given by [17]:

$$
\begin{aligned}
& \{\varepsilon\}=\left[S^{H}\right]\{\sigma\}+[d]^{T}\{H\} \\
& \{B\}=\left[\mu^{\sigma}\right]\{H\}+[d]\{\sigma\}
\end{aligned}
$$

where $\{\varepsilon\}$ and $\{\sigma\}$ are strain and stress respectively. $\left[S^{H}\right]$ is the elastic compliance under constant magnetic field and $\left[\mu^{\sigma}\right]$ is the permeability under constant stress. $[d]$ represents the magnetomechanical coupling coefficient. Equation 1 is the direct effect and Equation 2 is the converse effect.

For magnetoelectric sensor applications, a soft ferromagnetic material, with low hysteresis loss and steep slope in the B-H curve is desired since the material magnetization can be sensitively switched using application of low magnetic field amplitudes. Most commonly used and studied magnetostrictive materials are Terfenol-D [18], Galfenol [19] and Metglas ${ }^{\circledR}$. Terfenol-D exhibits the highest magnetostriction (>800 ppm) and the largest saturation field $(\sim 4 \mathrm{kOe})$ among these materials. Galfenol shows lower magnetostriction compared to Terfenol-D, however, it has a much higher permittivity. Metglas ${ }^{\circledR}$ shows the lowest magnetostriction ( $27 \mathrm{ppm})$, the smallest saturation field $(20 \sim 100 \mathrm{Oe})$ and the highest permeability $\left(\mu_{\mathrm{r}}>45,000\right)$. In this work, amorphous Metglas ${ }^{\circledR}$ 2605SA1 has been used as the magnetostrictive layer. Metglas $^{\circledR}$ is an iron, silicon and boron alloy $\left(\mathrm{Fe}_{85} \mathrm{~B}_{5} \mathrm{Si}_{10}\right)$ and has high bulk magnetostriction strain compared to similar materials, which is measured to be around $27 \mathrm{ppm}$. It is a good soft ferromagnetic material and has a high relative permeability that is around 45000 as cast [20].

The foremost challenge is the optimization of the deposition parameters of thin films of Metglas ${ }^{\circledR}$ since these have a significant impact on the magnetic properties of the thin film. To achieve this objective, a four gun ion beam deposition system has been designed and developed. The system consists of a vacuum chamber pumped using a turbomolecular pump and four ion guns from South Bay Technology Inc directed at the Metglas ${ }^{\circledR}$ target for deposition. Metglas ${ }^{\circledR}$ target was prepared by attaching three $25 \mu \mathrm{m}$ 
thick Metglas ${ }^{\circledR}$ sheets on an aluminum substrate. The sample was place a distance of $10 \mathrm{~cm}$ from the source. The deposition of the film was performed at a pressure of $60 \mu$ Torr and deposition rate of $\sim 1 \mathrm{~nm} / \mathrm{min}$.

$300 \mathrm{~nm}$ thick films were deposited and characterized for their BH loops. Quantum Design 1802 SQUID magnetometer with a field sweep range of $5 \mathrm{~T}$ at $305 \mathrm{~K}$ was used for these measurements. The samples are digitally imaged to extract the sample area and then sewn onto SQUID straws in order to obtain the B-H characteristics. Figure 1 shows the in-plane B-H loop of two different Metglas ${ }^{\circledR}$ 2605SA1 films, deposited on $2 \times 2 \times 1 \mathrm{~mm}$ glass slides using the same target under different ion-beam deposition conditions as described above. As it is observed from the figure, the hysteresis loss as well as the saturation magnetization can be controlled by applying in-plane magnetic field during the deposition. The relative permeability of the films are around 6500-7000, which is lower than the bulk values, but still shows reasonably high permeability. The coercive fields are 15 Oe and 5 Oe for film 1 and film 2 respectively. The saturation of magnetization of two films are $\sim 1 \mathrm{~T}$.

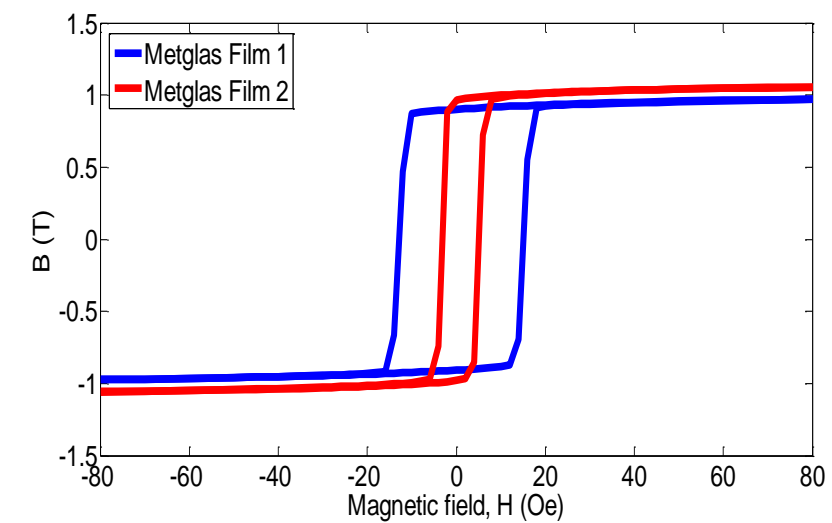

Figure 1: The BH curve of two different Metglas thin films. The film thicknesses are around $150 \mathrm{~nm}$.

\section{STRESS AND STRAIN SENSITIVITY OF QUARTZ}

It has been unambiguously observed that AT-cut quartz bulk acoustic wave resonators are highly sensitive to applied stresses and acceleration. As a result of applied transverse loading, both the thickness shear mode resonance frequency as well as the quality factor of the resonators change. The normalized frequency shift is denoted by $\Delta f l f_{0}$ where $\Delta f$ is the frequency shift due to applied stress, and $f_{0}$ is the fundamental resonance frequency of the quartz resonator. In literature, the resonance frequency shifts of circular AT-cut crystal plates subjected to transverse loading and radial loading are extensively studied by Lee [21] and Ballato [22]. A point load is applied to one end of the disk and resonance frequency shifts are measured experimentally as well as analytically modeled. As discussed in [23], the stress field in the quartz is very complex even though the plate is isotropic and rectangular. In the stress-strain relationship, the non-linear terms associated with the third-order elastic coefficients need to be included to solve for the exact frequency change. These assumptions will not be discussed within this paper, but a simplified equation estimating the frequency shift can be given by [23]:

$$
\begin{aligned}
& \frac{\Delta f}{f_{0}}=\frac{1}{2 C_{66}}\left(2 E_{1}^{(0)}+C_{661} E_{1}^{(0)}+C_{662} E_{2}^{(0)}+C_{663} E_{3}^{(0)}+C_{664} E_{4}^{(0)}\right) \\
& -\left(\frac{b^{2} /(3)^{1 / 2}}{\pi C_{66}}\left(C_{165} E_{5,1}^{(1)}+C_{561} E_{1,3}^{(1)}+C_{563} E_{3,3}^{(1)}\right)\right)
\end{aligned}
$$

where $E_{i}^{(0)}$ and $E_{i}^{(1)}$ are the zero order and first order strains respectively $(\mathrm{i}=1,2, \ldots, 6), C_{i j}$ and $C_{i j k}$ are the second order and third order elastic stiffness coefficients respectively, and $2 b$ is the thickness of the plate. A sensitivity of $\sim 166 \mathrm{~Hz} / \mathrm{N}$ for a 1 inch diameter crystal of AT-cut quartz has been reported [23]. As we will demonstrate in this work, that a much higher sensitivity can be achieved by micromachining quartz making it possible to achieve high magnetic field sensitivity.

\section{SENSOR FABRICATION}

An inverted mesa design circular quartz resonator with $350 \mu \mathrm{m}$ diameter front electrode and $22 \mu \mathrm{m}$ thickness was fabricated by dry etching of $100 \mu \mathrm{m}$ thick AT-cut quartz substrates. The details of the fabrication process can be found in reference [24]. This was followed by the deposition of an unpatterned $300 \mathrm{~nm}$ thick Metglas ${ }^{\circledR}$ film on the unetched side of the resonator using 4-gun ion beam deposition system at a rate of $\sim 1 \mathrm{~nm} / \mathrm{min}$. The deposited film has B-H loop characteristics similar to that of film 2 shown in Fig. 1. However, the circular shape of the resonator makes it mechanically stiff and difficult to strain the resonator either extensionally or flexurally. In order to increase the flexural bending sensitivity, a cantilever structure $500 \mu \mathrm{m}$ in length and 45 $\mu \mathrm{m}$ in width was ion-milled out of the circular resonator structure to result in a more compliant structure. In order to form and release the cantilever, Focused Ion Beam (FIB) milling was used. The FIB parameters are given in Table 1 . Since the cut time is $\sim 10$ hours, the quartz substrate showed considerable charge accumulation which resulted in the beam to vibrate sinusoidally. This has a negative effect on the dimension control of the cantilever. To avoid this problem, an electrically conductive probe is used to contact on the quartz surface. The final micromachined quartz cantilever resonator is shown in Fig. 2. As a result, a unimorph cantilever structure consisting of $22 \mu \mathrm{m}$ thick quartz and a $300 \mathrm{~nm}$ thick Metglas $^{\circledR}$ is formed. The $22 \mu \mathrm{m}$ thickness of the quartz results in a fundamental thickness shear frequency of $\sim 76.5 \mathrm{MHz}$. Governed by equation 1 , the induced strain due to magnetostriction is now expected to result in the shift of the resonance frequency as a function of the magnitude of the applied magnetic field and at a much higher sensitivity.

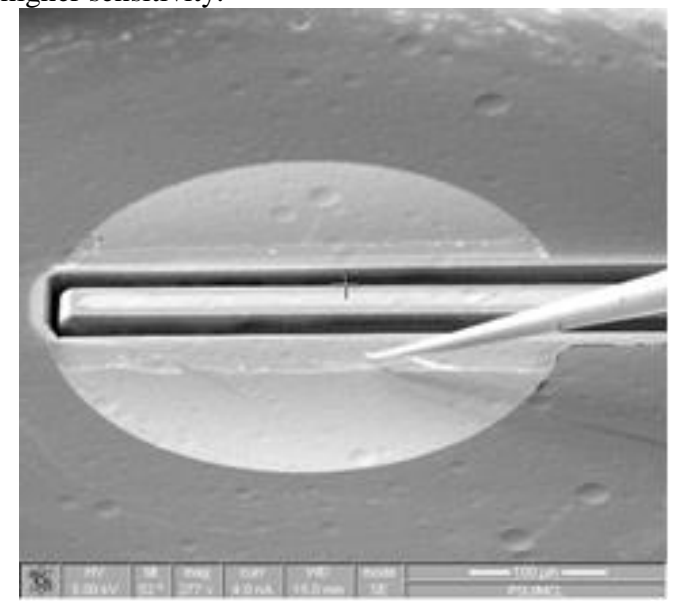

Figure 2: SEM picture while milling out the cantilever. The Omni probe is used in order to avoid charging.

Table 1: Focused Ion beam cut parameters

\begin{tabular}{|c|c|c|c|}
\hline & \multicolumn{3}{|c|}{ Focused Ion beam cut parameters } \\
\hline Cut time & Beam energy & Beam current & Dwell Time \\
\hline 10 hours & $30 \mathrm{kV}$ & $20 \mathrm{nA}$ & $1 \mu \mathrm{s}$ \\
\hline
\end{tabular}




\section{EXPERIMENTAL}

\section{Flexural Sensitivity: Thermal Test}

In order to determine the sensitivity of the quartz resonator to strain input we performed a thermal unimorph test. Since the sensor cantilever is a bilayer structure consisting of quartz and Metglas $^{\circledR}$ layers, the temperature coefficient of expansion mismatch between the two layers will result in net out of plane moment. Furthermore, for small temperature excursions of less than $10{ }^{\circ} \mathrm{C}$, the shift in AT cut quartz is very minimal. In fact, the frequency shift is on the order of ppm [25]. Thus, any resulting frequency change can be attributed to the resulting thermal strain in the device structure. Since, thermal actuation is theoretically very well understood and thermal expansion coefficients of the two materials are well characterized and known, the experimentally measured frequency shift in the resonance frequency can be easily related to the theoretical deflection from the bilayer microactuator. The bilayer cantilever curvature due to a temperature difference $\Delta T$ is given by [26]:

$$
k=\frac{6 b_{1} b_{2} E_{1} E_{2} t_{1} t_{2}\left(t_{1}+t_{2}\right)\left(\alpha_{2}-\alpha_{1}\right) \Delta T}{\left(b_{1} E_{1} t_{1}^{2}\right)^{2}+\left(b_{2} E_{2} t_{2}^{2}\right)^{2}+2 b_{1} b_{2} E_{1} E_{2} t_{1} t_{2}\left(2 t_{1}^{2}+3 t_{1} t_{2}+2 t_{2}^{2}\right)}
$$

where subscript 1 and 2 refer to material properties of quartz and Metglas ${ }^{\circledR}$ layers respectively. The parameters $b, E, t$ are the width, elastic modulus, and thickness respectively. $\alpha$ is the thermal expansion coefficient and $\Delta T$ is the temperature difference. The curvature is related to tip deflection, $d$ as $d=k L^{2} / 2$, where $L$ is the length of the cantilever. Figure 3 plots the experimentally measured frequency shift vs the associated phase shift (y-axis1) of the cantilever resonator for five $\Delta T$ values. The right hand side axis is the corresponding calculated tip deflection. The sensitivity is the slope of Fig. 3, which indicates $78 \mathrm{pm} / \mathrm{Hz}$. This sensitivity is encouraging to detect extremely small perturbations occurring within the quartz as a result of magnetostrictive bending of the unimorph.

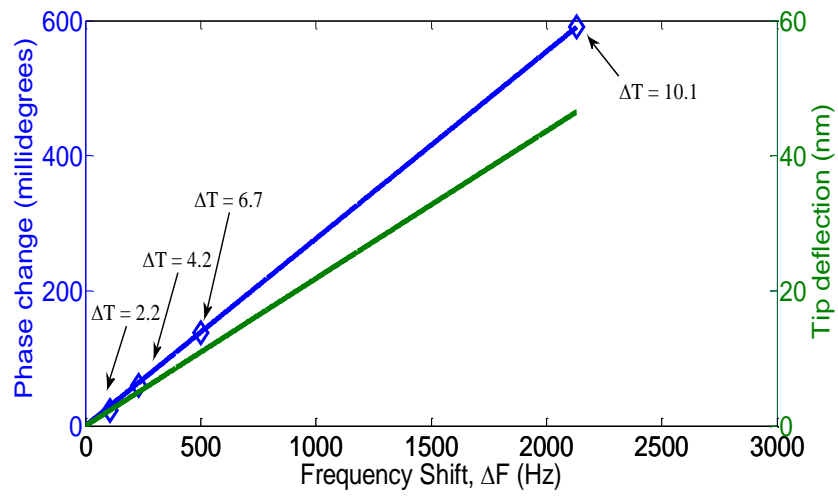

Figure 3: The peak to peak phase change and calculated tip deflection $v$ s. the frequency shift, $\Delta F$.

\section{Magnetic Tests}

The magnetic experimental setup consists of a Helmholtz coil with a diameter of $15 \mathrm{~cm}$ and 140 turns $(\mathrm{N}=140)$. Applied magnetic field is precisely controlled via the current through the coil. Quartz microcantilever impedance characteristics were monitored in real-time using an Agilent E5061B network analyzer. The S-parameters are converted into impedance parameters using a Labview $^{\circledR}$ program. The phase of the resonator was measured at a fixed frequency corresponding to the maximum slope of the phasefrequency curve and recorded as a function of the applied magnetic field.

The first cantilever resonator we tested was an AT-cut quartz resonator with a thickness of $\sim 37 \mu \mathrm{m}$, length of $200 \mu \mathrm{m}$ and a width of $50 \mu \mathrm{m}$. The Metglas ${ }^{\circledR}$ was $150 \mathrm{~nm}$ thick. The device was placed in the middle of the Helmholz coil set-up and a square wave magnetic field with a frequency of $0.075 \mathrm{~Hz}$ corresponding to a cycle time of $13.33 \mathrm{~s}$ was applied. The peak-to-peak change in the susceptance of the resonator at resonance in response to the applied magnetic field was measured and is shown in Fig. 4. From the slope of the curve the sensitivity of the device can be given to be $0.095 \mu \mathrm{S} / \mu \mathrm{T}$.

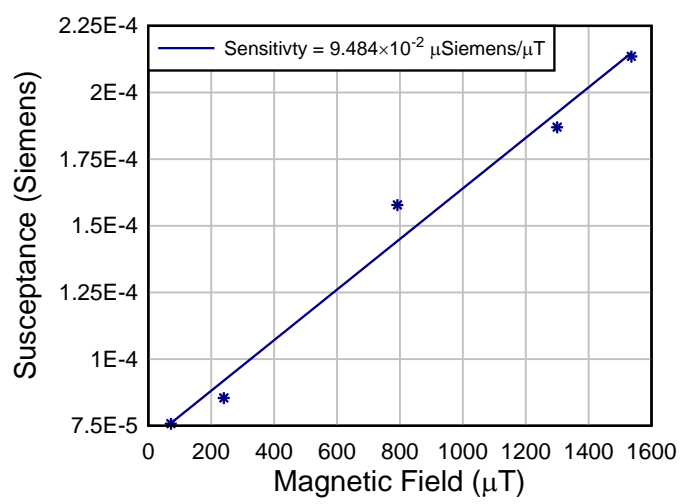

Figure 4: Sensitivity of quartz resonator susceptance at resonance to magnetic field is measured and shows a linear dependence.

Figure 5 shows the response of the $76 \mathrm{MHz}, 500 \mu \mathrm{m}$ long cantilever sensor to an applied square-wave, peak-to-peak magnetic field of $1.5 \mu \mathrm{T}$. The $x$-axis is time and the $y$-axis corresponds to phase shift of the resonator. For the resonator any of the impedance and admittance characteristics at resonance can be monitored as a function of the applied magnetic field. To ensure maximum sensitivity the frequency needs to be set at the maximum of the slope of the frequency vs quantity of measurement. Based on the signal-to-noise ratio performance nT range of sensitivity can be obtained.

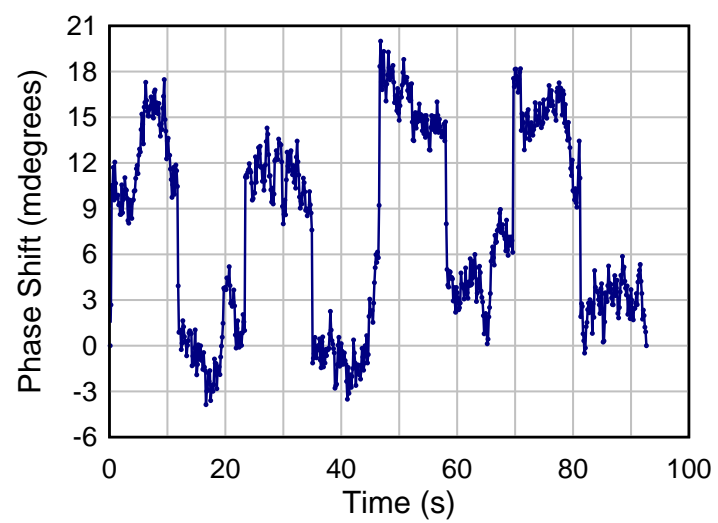

Figure 5: The phase change as a result of the applied ac magnetic field of $1.5 \mu T$.

\section{DISCUSSION}

In this paper, the concept detecting magnetic fields by monitoring the induced changes in the at-resonance impedance and admittance characteristics of AT-cut quartz resonators has been explored. It is observed that the bending of the cantilever due to magnetostrictive strain of the magnetic layer affects the at- 
resonance impedance and admittance characteristics of the resonator very sensitively. Using thermal unimorph bending performance of the device a sensitivity of $78 \mathrm{pm} / \mathrm{Hz}$ was experimentally determined. The response of the resonator phase to an applied field of $1.5 \mu \mathrm{T}$ was obtained and the signal-to-nose sensitivity of the device shows that nanoTesla range sensitivity can be obtained by further optimizing the thicknesses, dimensions, and the cut of the quartz crystal. Optimization of the deposited Metglas ${ }^{\circledR}$ films are also expected improve the sensitivity of the device further.

\section{ACKNOWLEDGEMENTS}

This work was supported in part by National Science Foundation (NSF) under the grant \# 1305653 The use of facilities at the PSU Site of the NSF National Nanotechnology Infrastructure Network (NNIN) under Agreement 0335765 is acknowledged. GH acknowledges partial support from Scientific and Technical Research Council of Turkey (TUBITAK) for a 2213-International $\mathrm{PhD}$. Fellowship Program. GH also thanks to Transducers Research Foundation for the travel support.

\section{REFERENCES}

[1] P. Ripka, Ed., Magnetic Sensors and Magnetometers. Boston: Artech House Publishers, 2001, p.^^p. Pages.

[2] S. Dong, "Ultrahigh magnetic field sensitivity in laminates of TERFENOL-D and $\mathrm{Pb}(\mathrm{Mg} 1 / 3 \mathrm{Nb} 2 / 3) \mathrm{O} 3$ ?PbTiO3 crystals," Appl. Phys. Lett., vol. 83, p. 2265, 2003.

[3] J. Zhai, Z. Xing, S. Dong, J. Li, and D. Viehland, "Detection of pico-Tesla magnetic fields using magneto-electric sensors at room temperature," Applied Physics Letters, vol. 88, p. 062510, 2006.

[4] J. Lenz and S. Edelstein, "Magnetic sensors and their applications," Sensors Journal, IEEE, vol. 6, pp. 631649, 2006.

[5] R. L. Fagaly, "Superconducting quantum interference device instruments and applications," Review of Scientific Instruments, vol. 77, p. 101101, 2006.

[6] J. P. Wikswo, Jr., "SQUID magnetometers for biomagnetism and nondestructive testing: important questions and initial answers," Applied Superconductivity, IEEE Transactions on, vol. 5, pp. 74-120, 1995.

[7] I. K. Kominis, T. W. Kornack, J. C. Allred, and M. V. Romalis, "A subfemtotesla multichannel atomic magnetometer," Nature, vol. 422, pp. 596-599, 2003.

[8] I. M. Savukov and M. V. Romalis, "NMR Detection with an Atomic Magnetometer," Physical Review Letters, vol. 94, p. 123001, 2005.

[9] J. E. Lenz, "A review of magnetic sensors," Proceedings of the IEEE, vol. 78, pp. 973-989, 1990.

[10] F. Li, R. Misra, F. Zhao, Y. Wu, P. Schiffer, Q. M. Zhang, S. Tadigadapa, and S. Datta, "Magnetoelectric Resonant Gate Transistor with NanoTesla Sensitivity," Journal of Microelectromechanical Systems, vol. In preparation, 2012.

[11] H. Gokhan, "A novel magnetometer employing magnetoviscous effect of ferrofluids," Hilton Head Technical Digest, pp. 42-45, 20122012.

[12] S. Dong, "Giant magnetoelectric effect (under a dc magnetic bias of 2?Oe) in laminate composites of FeBSiC alloy ribbons and $\mathrm{Pb}(\mathrm{Zn} 1 / 3, \mathrm{Nb} 2 / 3) \mathrm{O} 3$ ?7\%PbTiO3 fibers," Appl. Phys. Lett., vol. 91, p. 022915, 2007.

[13] S. Dong, J. Zhai, J. Li, and D. Viehland, "Near-ideal magnetoelectricity in high-permeability magnetostrictive/piezofiber laminates with a (2-1) connectivity," Applied Physics Letters, vol. 89, p. 252904, 2006.

[14] C.-W. Nan, M. I. Bichurin, S. Dong, D. Viehland, and G. Srinivasan, "Multiferroic magnetoelectric composites: Historical perspective, status, and future directions," Journal of Applied Physics, vol. 103, p. 031101, 2008.

[15] F. Zhao, N. Mokhariwale, L. Feng, S. Datta, and Q. M. Zhang, "Magnetoelectric Sensors With Directly Integrated Charge Sensitive Readout Circuit\&\#x2014; Improved Field Sensitivity and Signalto-Noise Ratio," Sensors Journal, IEEE, vol. 11, pp. 2260-2265, 2011

[16] R. Brendel, "Influence of a magnetic field on quartz crystal resonators," Ultrasonics, Ferroelectrics and Frequency Control, IEEE Transactions on, vol. 43, pp. 818-831, 1996.

[17] M. J. Dapino, R. C. Smith, and A. B. Flatau, "Model for the $\Delta \mathrm{E}$ effect in magnetostrictive transducers," 2000, pp. 174-185.

[18] A. E. Clark and D. Crowder, "High temperature magnetostriction of $\quad \mathrm{TbFe}<\mathrm{inf}>2</ \mathrm{inf}>$ and Tb<inf $>.27</$ inf $>$ Dy $<$ inf $>.73</$ inf $>$ Fe $<$ inf $>2</$ inf $>$," Magnetics, IEEE Transactions on, vol. 21, pp. 19451947, 1985.

[19] T. Takahashi, T. Okazaki, and Y. Furuya, "Improvement in the mechanical strength of magnetostrictive ( $\mathrm{Fe}-\mathrm{Ga}-$ $\mathrm{Al})-\mathrm{X}-\mathrm{C}(\mathrm{X} \& \# \mathrm{xa} 0 ;=\& \# \mathrm{xa} 0 ; \mathrm{Zr}, \mathrm{Nb}$ and $\mathrm{Mo})$ alloys by carbide precipitation," Scripta Materialia, vol. 61, pp. 57, 2009

[20] M. C. Products. (2014, 03/25/2014). Metglas ${ }^{\circledR} 2605 S A 1$ \& 2605HB1M Magnetic Alloy properties. Available: http://www.metglas.com/products/magnetic materials/26 05SA1.asp

[21] P. C. Y. Lee, Y. S. Wang, and X. Markenscoff, "Effects of Initial Bending on the Resonance Frequencies of Crystal Plates," in 28th Annual Symposium on Frequency Control. 1974, 1974, pp. 14-18.

[22] A. D. Ballato, "Effects of Initial Stress on Quartz Plates Vibrating in Thickness Modes," in 14th Annual Symposium on Frequency Control. 1960, 1960, pp. 89114.

[23] P. C. Y. Lee, Y. S. Wang, and X. Markenscoff, "Nonlinear effects of initial bending on the vibrations of crystal plates," The Journal of the Acoustical Society of America, vol. 59, pp. 90-96, 1976.

[24] P. Kao, D. Allara, and S. Tadigadapa, "Characterization of viscoelastic properties of adsorbed biomolecules and biomolecular assemblies with high frequency micromachined quartz resonators," Sensors and Actuators B: Chemical, vol. 142, pp. 406-411, November 5, 20092009.

[25] "Basic Technology of Quartz resonators," ed, 2014.

[26] W.-H. Chu, M. Mehregany, and R. L. Mullen, "Analysis of tip deflection and force of a bimetallic cantilever microactuator," Journal of Micromechanics and Microengineering, vol. 3, p. 4, 1993.

\section{CONTACT}

*Srinivas Tadigadapa, Tel: +1-814-865-2730; sat10@psu.edu 